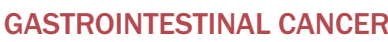

\section{Combine and conquer}

Despite the fact that up to $10 \%$ of colorectal cancers (CRC) have mutations in the Val600 codon of BRAF, the clinical response to treatment with the $\mathrm{V} 600 \mathrm{E}$ B-Raf targeted agent vemurafenib has not been encouraging. Researchers based at Roche (the developing company of vemurafenib) and led by Fei Su have, therefore, been looking for combination therapies to improve the efficacy of this targeted agent in the CRC setting.

Su explains the rationale of the preclinical research "we started out by evaluating the antitumor activity of vemurafenib in CRC cell lines and in mice bearing CRC xenografts, when administered as monotherapy and in combination with standard of care agents." Along the way they identified a CRC cell line (RKO cells) that was de novo resistant to vemurafenib treatment-an ideal tool for investigating the mechanisms of innate resistance and to identify combination therapies. Su comments that "one of the potential mechanisms for resistance was parallel activation of the PI3K/Akt pathway." This hypothesis was supported by improved efficacy for vemurafenib in the RKO model when combined with an Akt inhibitor.

The researchers also tested triplet therapies in the mouse xenograft models; a technique described by Su as "very difficult." But, using this method it was possible to show that the addition of vemurafenib to chemotherapy regimens (capecitabine and bevacizumab or cetuximab and irinotecan) improved survival in these models.

These preclinical data are obviously only an indication of how these combinations might perform in the clinic. However, they indicate that vemurafenib might have a role in CRC.

\section{Rebecca Kirk}

Original article Yang, $\mathrm{H}$ et al. Antitumor activity of BRAF inhibitor vemurafenib in preclinical models of BRAF-mutant colorectal cancer. Cancer Res. doi:10.1158/0008-5472.CAN-11-2941 